\title{
Investigation of Species Specificity Using Nine PCR-Based Human STR Systems
}

\author{
REFERENCE: Crouse, C. A. and Schumm, J., "Investigation of \\ Species Specificity Using Nine PCR-Based Human STR \\ Systems," Journal of Forensic Sciences, Vol. 40, No. 6, November \\ 1995, pp. 952-956.
}

\begin{abstract}
Several eukaryotic genomes contain polymorphic markers consisting of trimeric and tetrameric short tandem repeats (STR). Recent reports have demonstrated the variability of short tandem repeat (STR) polymorphisms at a variety of loci among several human population groups. Currently, there are nine commercially available STR PCR systems from Promega Corporation that may be utilized for human identification. We report here the analysis of 23 different species DNA's using these nine STR primer systems to assess their specificity for human euchromatin. The STR systems tested include, CSF1PO, TPOX, THO1, HPRTB, FESFPS, vWF and F13A01 as single systems and as triplex systems (CSF1PO/ TPOX/THO 1 and HPRTB/FESFPS/vWF). There were no STR PCR products observed for seventeen of the twenty-three species regardless of the STR system. Amplified STR fragments were detected in rhesus DNA for CSF1PO, TPOX and HPRTB systems. STR PCR products were detected for human, gorilla, chimpanzee, and orangutan DNAs using eight of the nine systems. FESFPS primers did not amplify DNA fragments from any of the species tested. Most of the STR PCR products detected from primate DNAs electrophoretically migrated outside of the human allelic ladder fragments and as a result, allele designations were not possible.
\end{abstract}

KEYWORDS: forensic science, polymerase chain reaction, short tandem repeats

The greater majority of eukaryotic genomic sequences have been shown to contain repetitive sequences which are stably inherited from generation to generation (1). These tandemly reiterated sequences are highly polymorphic and are thought to bear an evolutionary significance with regard to DNA fidelity. The organization of repetitive DNA sequences is based upon the degree of repeatability with satellite DNA demonstrating the highest level of repeat sequences, moderately repetitive sequences within minisatellite and microsatellite DNA sequences, transposable elements that are moderately repetitive and mobile, and short tandem repeats which consist of 2-7 nucleotide repeated sequences in a tandem array $[1,2]$. Recent reports have evaluated a battery of STR systems in the human population [3-7] and have concluded that STR systems are a invaluable investigative instrument for the purpose of personal identification.

The most recent DNA analytical tests which have been added to paternity, clinical and forensic laboratories are the use of the

Received for publication $20 \mathrm{Jan}$. 1995; revised manuscript received 4 April 1995; accepted for publication 25 April 1995.

'Senior Forensic Scientist, Palm Beach County Sheriff's Crime Laboratory, West Palm Beach, FL.

${ }^{2}$ Director, Research and Development-DNA Typing, Promega Corporation, Madison, WI.
PCR-based STR systems. For forensic laboratories, TWGDAM [8] and NRC guidelines [9] suggest it is important for each laboratory to validate DNA systems before implementation on case work, or to cite the literature for specific validation studies.

One of the recommendations is the determination of species specificity for new PCR primer systems. In this report, we have validated the species specificity of nine commercially available human STR systems on 23 species DNAs including primate and non-primate species. We have determined that for the species tested, no amplified products are detected for non-primate species whereas the five primate species generally exhibit amplified fragments for most of the nine human-derived STR primer pairs.

\section{Materials and Methods}

\section{DNA Sources, Extraction, Quantification}

DNA was organically extracted by standard protocol [10]. Primate DNA was extracted from whole blood collected from orangutan, chimpanzee, and gorilla species from the Miami Metro Zoo. Dr. Carolyn Cray, Associate Professor, University of Miami School of Veterinarian Medicine generously provided whole blood from frog (Xenopus), mouse (Balb/c), fox, raccoon, dolphin, sea lion, otter, ferret, lynx, Rhesus monkey and lymphocytes from the damsel fish. Commercial DNAs were purchased from ClonTech (Palo Alto, CA) including canine, bovine, chicken, Rhesus monkey, and porcine samples. Feline DNA was organically extracted from whole blood and was a generous gift from Dr. A. Angel, Animal Hospital of the Palm Beaches.

DNA extracts were quantified by one of two methods: 1) $0.7 \%$ agarose gel electrophoresis with Lambda HindIII as the mass standard, or 2) spectrophotometrically.

Amplification and Gel Electrophoresis-Amplification of STR alleles using Promega's individual primer sets or the STR Multiplex Systems was performed as per manufacturer's protocol. Briefly, amplification conditions were conducted in a $25 \mu \mathrm{L}$ reaction mixture containing $2.5 \mu \mathrm{L} \mathrm{10X} \mathrm{STR} \mathrm{buffer,} 2.5 \mu \mathrm{L} 10 \mathrm{X}$ primer pairs, $0.15 \mu \mathrm{L}(0.75$ Units) of AmpliTaq polymerase (Perkin Elmer, Alameda, CA) and $25 \mathrm{ng}$ of DNA. K562 DNA was used as a positive control. The PCR cycling parameters using the Perkin Elmer GAP 9600 was as per manufacture's suggestion. All primate species were amplified twice for each STR system. Verification of amplified products ( $9 \mu \mathrm{L} /$ reaction), was performed by electrophoreses on a $3 \%$ MetaPhor agarose gel (FMC, Rockland, ME) at 150 volts for 25 minutes. The STR amplified fragments were visualized using ethidium bromide (AMRESCO, Solon, $\mathrm{OH}$ ). The $1 \mathrm{~Kb}$ DNA Ladder (BRL, Gaithersburg, MD) was used as a molecular weight standard. Gels were photographed using a Polaroid MP4+ camera. 
The STR alleles were separated on the SA32 vertical gel apparatus (BRL, Bethesda, MD) using a $4 \%$ denaturing polyacrylamide gel (PAG). Post-polymerization, the PAG was pre-run for 60 minutes at 40 watts. The DNA samples and STR ladders were prepared by placing $2.5 \mu \mathrm{L}$ amplified product and $2.5 \mu \mathrm{L}$ STR $2 \mathrm{X}$ Loading Solution at $95^{\circ} \mathrm{C}$ for two minutes followed by a quick

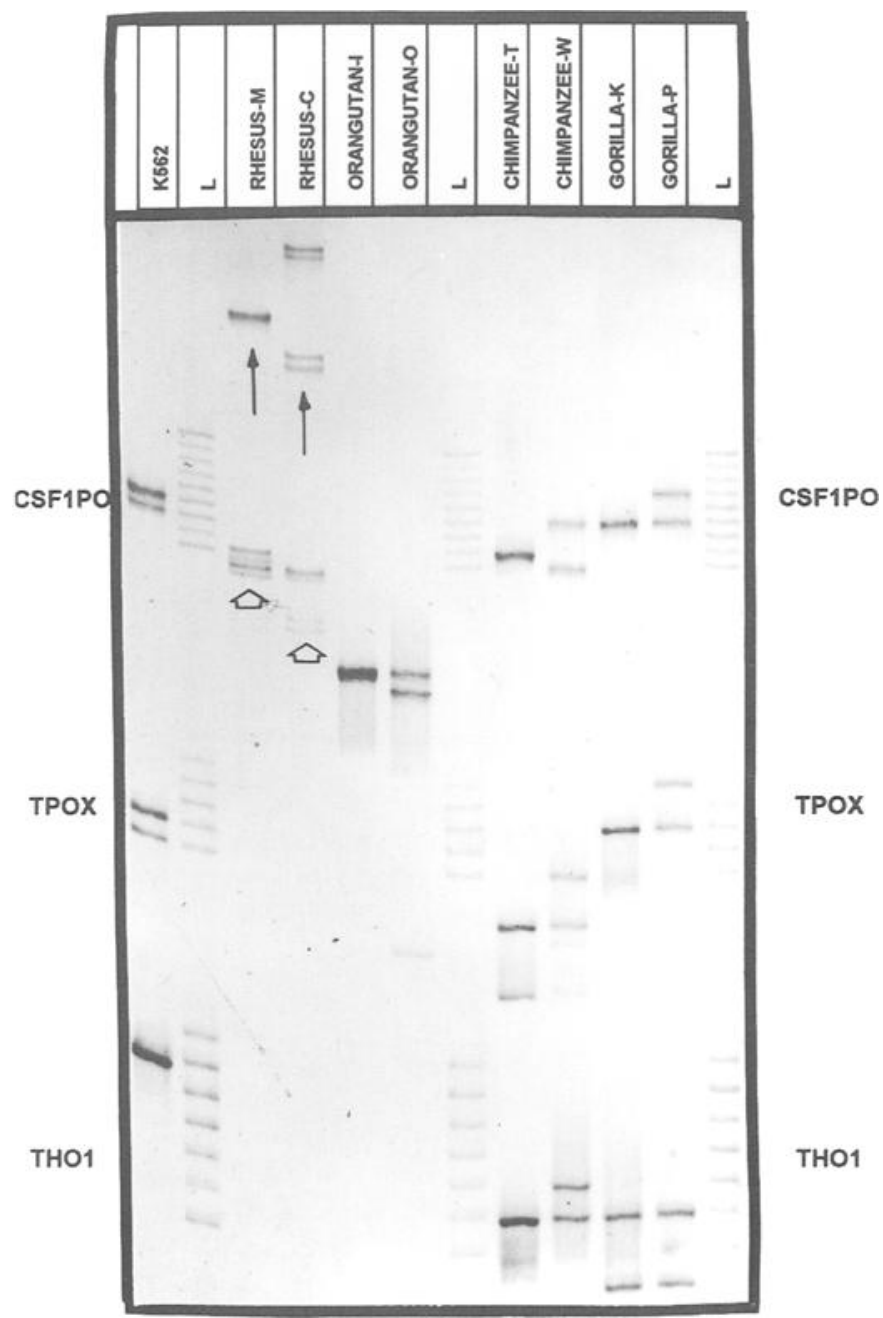

FIG. 1-Amplification and polyacrylamide gel electrophoresis of primate DNA samples using the GenePrint CTT triplex system. DNA samples from the species indicated above were amplified as described in the Materials and Methods. Lanes labeled " $L$ " contain the CSFIPO-TPOX-THOI allelic ladder mix. Closed and open arrow's indicate the products generated by amplification at the CSFIPO and TPOX loci, respectively. submersion on ice. The $0.4 \mathrm{~mm}$ doublefine sharkstooth comb (BRL, Bethesda, MD) or $0.4 \mathrm{~mm}$ fixed well comb (Owl Scientific, Boston, MA) was used to load $3 \mu \mathrm{L}$ of each sample. The samples were electrophoresed for approximately 80 minutes at 40 watts. STR fragments were visualized using the DNA Silver Staining Kit (Promega, Madison, WI) as per protocol. Archival copies of stained PAGs were made using Kodak Electrophoresis Duplicating Film (EDF).

\section{Results and Discussion}

The commercially available STR systems utilized for these studies are described in Table 1. All of these STR loci have been well-characterized with regard to chromosomal location, repeat sequence, and allelic polymorphisms [2-7,11-16].

The intent of this report is to evaluate the species specificity of nine STR systems including the monosystems CSF1PO, TPOX, THO1, HPRTB, FESFPS, vWF and F13A01 as well as two STR triplex systems, CSF1PO/TPOX/THO1 and HPRTB/FESFPS/ vWF. No PCR products were detected in 17 of the 23 species tested including frog (Xenopus), mouse (Balb/c), fox, raccoon, dolphin, sea lion, otter, ferret, lynx, damsel fish, macaw, shark, snake, canine, bovine, chicken, porcine and feline for the nine systems analyzed (data not shown).

The only species demonstrating any amplified STR products using the systems described were of primate origin. Figure 1 shows the results of amplifying two samples each from rhesus, orangutan, chimpanzee and gorilla DNA using the CTT multiplex system. Rhesus-M DNA showed one band within the CSF1PO allelic ladder and three bands below the CSF1PO ladder. Rhesus-C showed four bands immediately below this same ladder. Further analysis of these two DNAs using the individual CSF1PO and TPOX monosystems (Fig. $2 A$ and $2 B$ ) demonstrated that all of the rhesus fragments detected well above the higher molecular weight CSF1PO human alleles are products from CSF1PO amplification (Figs. 1 and 2, closed arrows) and the amplified DNA shown near the lower molecular weight CSF1PO allelic ladder are the result of amplification with the TPOX primers (Figs. 1 and 2, open arrows). No rhesus PCR products were detected using THO1 primers in either the triplex system (Fig. 1) or the THO1 monosystem (data not shown). The only amplified orangutan DNA products were detected between the CSF1PO and TPOX ladder (Fig. 1) and were attributed to the CSF1PO primer pair (Fig. 2A). TPOX primers will weakly amplify orangutan DNA although the bands are detected below the TPOX allelic ladder (Figs. 1 and $2 B$ ).

The two chimpanzee DNA samples show amplified products within the range of all three CTT loci. Each chimpanzee sample exhibits different band patterns for each of the STR systems tested (Fig. 1). The CSF1PO alleles detected in the chimpanzee-T and

TABLE 1-STR locus specific information.

\begin{tabular}{lcccc}
\hline $\begin{array}{c}\text { GenePrint } \\
\text { STR System } \\
\text { locus }\end{array}$ & $\begin{array}{c}\text { Chromosomal } \\
\text { location }\end{array}$ & $\begin{array}{c}\text { Repeat } \\
\text { Sequence } \\
5^{\prime} \rightarrow 3^{\prime}\end{array}$ & $\begin{array}{c}\text { Allelic Ladder } \\
\text { Size Ranges } \\
\text { (bases) }\end{array}$ & $\begin{array}{c}\text { Total \# of } \\
\text { Alleles in } \\
\text { ladder }\end{array}$ \\
\hline CSF1PO & $5 q 33.3-34$ & AGAT & $299-323$ & $7,8,9,10,11,12,13,14,15$ \\
TPOX & 2 p13 & AATG & $232-248$ & $8,9,10,11,12$ \\
THO1 & 11 p15.5 & AATG & $179-203$ & $5,6,7,8,9,10,11$ \\
HPBRT & Xq26 & AGAT & $259-303$ & $6,7,8,9,10,11,12,13,14,15,16,17$ \\
FESFPS & $15 q 25-q$ ter & AAAT & $222-250$ & $7,8,9,10,11,12,13,14$ \\
vWF & 12 p12-pter & AGAT & $139-167$ & $13,14,15,16,17,18,19,20$ \\
F13A01 & 6p24-25 & AAAG & $169-185$ & $4,5,6,7,8,9,11,12,13,14,15,16$ \\
\hline
\end{tabular}




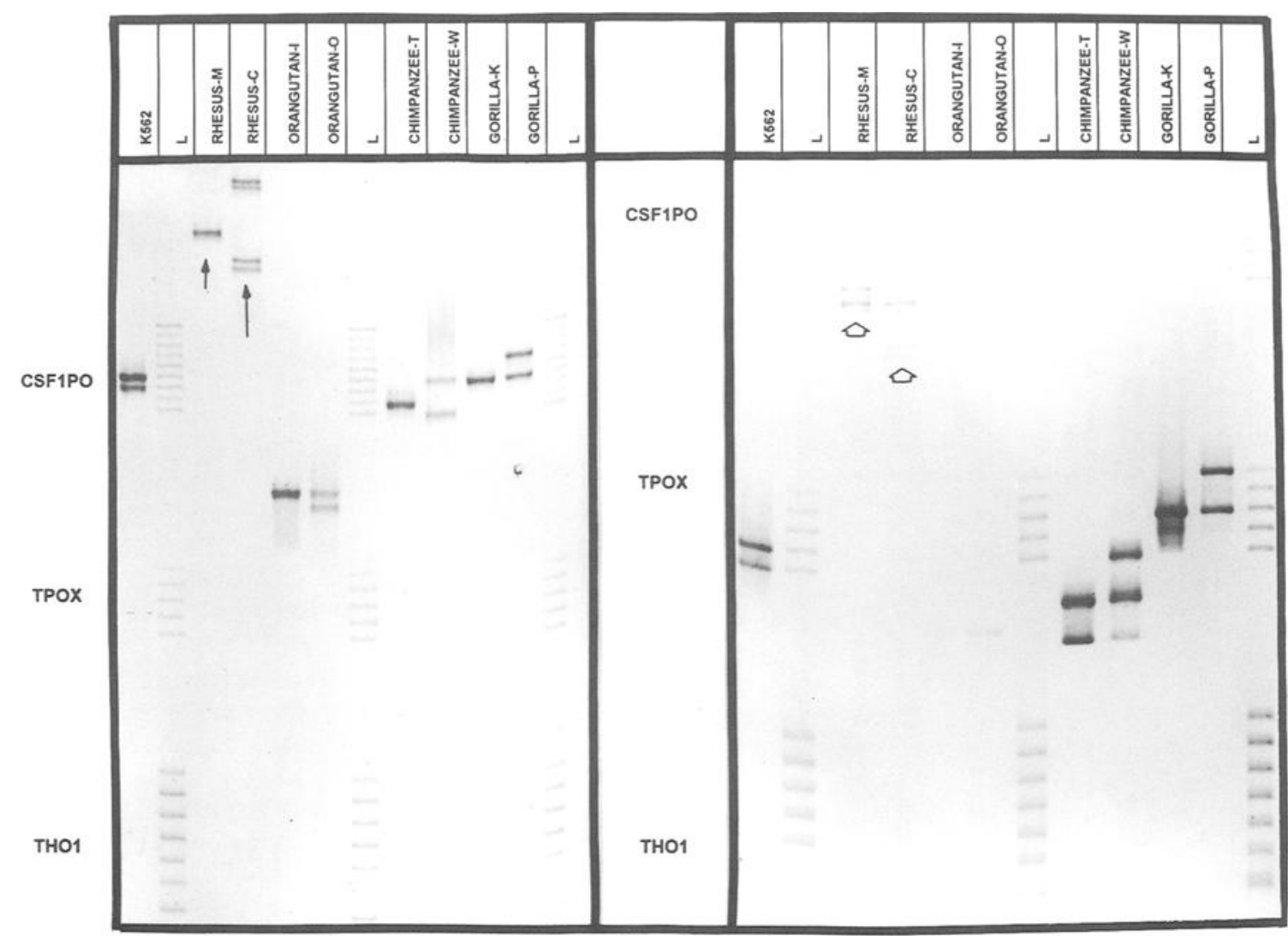

$2 A$

2B

FIG. 2-Amplification of primate DNA samples using primer pairs specific for the CSFIPO loci (2A) and the TPOX loci (2B). DNA samples from the species indicated above were amplified as described in the Materials and Methods. Closed and open arrows indicate the products generated by amplification at the CSFIPO and TPOX loci, respectively. The CSFIPOITPOXITHOI combination allelic ladder was electrophoresed with these samples in order to evaluate amplified products.

Chimpanzee-W samples are " 8 " and " 7,11 ," respectively (Fig. $2 A)$. It is not known if the " 8 " allele for the chimpanzee-T is homozygous or if the second allele was not amplified due to primer specificity. TPOX alleles for the chimpanzee-T migrate below the TPOX allelic ladder and as a result, no allele determination can be made (Fig. $2 B$ ). Chimpanzee-W can be assigned the TPOX " 8 " allele but the second allele migrates below the ladder as seen with chimpanzee-T. The chimpanzees exhibit ampiified THO1 products aligning with alleles " 6 " and " 6,7 " for chimpanzee-T and $W$, respectively (Fig. 1).
The gorilla DNAs also amplified STR products for all three CTT primer sets (Fig. 1). The CSF1PO alleles are "10" for gorilla-K and " 10,12 " for gorilla-P (Figs. 1 and $2 A$ ). The TPOX primers amplified products coincident with TPOX alleles " 10 " and " 10,12 " for gorilla-K and P. These DNAs also showed amplified THO1 products that were identical for both gorilla samples. One of the alleles migrated adjacent to the THO1 " 6 " allele but the second allele migrated below the THO-1 ladder and could not be given an allelic designation.

All species DNAs were also analyzed for amplified products

TABLE 2-Summary of primates STR band patterns.

\begin{tabular}{|c|c|c|c|c|c|c|c|c|c|c|c|c|c|c|}
\hline & \multicolumn{2}{|c|}{ CSFIPO } & \multicolumn{2}{|c|}{ TPOX } & \multicolumn{2}{|c|}{ THOI } & \multicolumn{2}{|c|}{ HPRTB } & \multicolumn{2}{|c|}{ FESFPS } & \multicolumn{2}{|c|}{ vWF } & \multicolumn{2}{|c|}{ F13A01 } \\
\hline & $\begin{array}{c}\text { \# of } \\
\text { bands }\end{array}$ & Alleles & $\begin{array}{l}\text { \# of } \\
\text { bands }\end{array}$ & Alleles & $\begin{array}{c}\text { \# of } \\
\text { bands }\end{array}$ & Alleles & $\begin{array}{c}\text { \# of } \\
\text { bands }\end{array}$ & Alleles & $\begin{array}{c}\text { \# of } \\
\text { bands }\end{array}$ & Alleles & $\begin{array}{c}\text { \# of } \\
\text { bands }\end{array}$ & Alleles & $\begin{array}{c}\text { \# of } \\
\text { bands }\end{array}$ & Alleles \\
\hline Rhesus-M & 1 & $>A L$ & 4 & $>A L$ & $\mathrm{X}$ & $\mathrm{X}$ & 2 & $12,>\mathrm{AL}$ & $\mathrm{X}$ & $\mathrm{X}$ & $\mathrm{X}$ & $\mathrm{X}$ & $\mathrm{X}$ & $\mathrm{X}$ \\
\hline Rhesus-C & 4 & $>A L$ & 4 & $>A L$ & $\mathrm{X}$ & $\mathrm{X}$ & 1 & $>A L$ & $\mathrm{X}$ & $\mathrm{X}$ & $\mathrm{X}$ & $\mathrm{X}$ & $\mathrm{X}$ & $X$ \\
\hline Orangutan-I & 1 & $<\mathrm{AL}$ & 1 & $<\mathrm{AL}$ & $\mathrm{X}$ & $\mathrm{X}$ & $\mathrm{X}$ & $\mathrm{X}$ & $\mathrm{X}$ & $\mathrm{X}$ & $\mathrm{X}$ & $\mathrm{X}$ & 1 & 12 \\
\hline Orangutan-O & 2 & $<\mathrm{AL}$ & 1 & $<\mathrm{AL}$ & $\mathrm{X}$ & $\mathrm{X}$ & 1 & 7 & $X$ & $\mathrm{X}$ & $\mathrm{X}$ & $\mathrm{X}$ & 1 & 12 \\
\hline Chimpanzee- $\mathrm{T}$ & 1 & 6 & 2 & $<\mathrm{AL}$ & 1 & 6 & 2 & 12,13 & $\mathrm{X}$ & $\mathrm{X}$ & 2 & $<\mathrm{AL}, 6$ & 1 & 6 \\
\hline Chimpanzee-W & 2 & 7,10 & 2 & $<\mathrm{AL}$ & 2 & 6,7 & 1 & 11 & $\mathrm{X}$ & $\mathrm{X}$ & 2 & $<\mathrm{AL}$ & 1 & 6 \\
\hline Gorilla-K & 1 & 10 & 1 & 10 & 2 & $6,<\mathrm{AL}$ & 2 & 11,12 & $\mathbf{X}$ & $\mathrm{X}$ & 2 & $\mathrm{OL}$ & 1 & 4 \\
\hline Gorilla-P & 2 & 10,12 & 2 & 10,12 & 2 & $6,<\mathrm{AL}$ & 1 & 12 & $X$ & $\mathrm{X}$ & 2 & OL & 1 & 4 \\
\hline
\end{tabular}

NOTE: The number and relative allelic ladder (AL) location of the primate DNA amplified products for six of the STR primer pairs is summarized. Amplified products are noted as electrophoretically migrating above the AL ( $>A L$ ), below the AL ( $\angle A L)$, within the AL but not adjacent to an AL fragment (OL) or no fragments detected (X). If amplified products were detected adjacent to AL fragments they were assigned the allelic repeat number. 


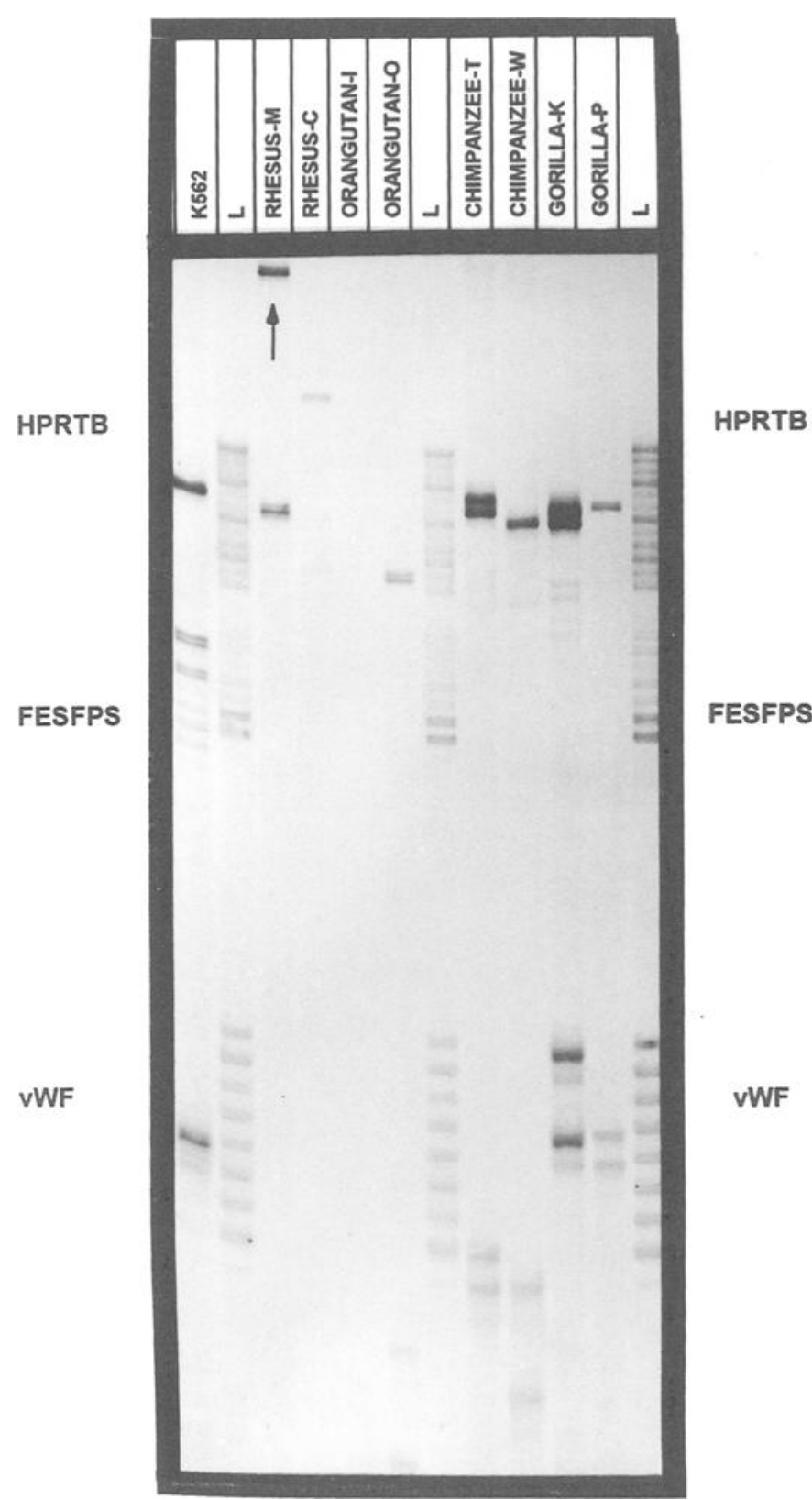

FIG. 3-Amplification and polyacrylamide gel electrophoresis of primate DNA samples using the GenePrint HPRTB/FESFPS/vWF triplex. DNA samples from the species indicated above were amplified as described in the Materials and Methods. Lanes labeled " $L$ " contain the HPRTBFESFPS-vWF allelic ladder mix.

using the second triplex and individual monosystems which includes the loci HPRTB, FESFPS, vWF (Fig. 3). The same seventeen species which were negative for amplified products using the systems described above, were also negative when the second triplex and monosystems were tested (see above). The primate DNAs were the only species to demonstrate STR amplified fragments for this second triplex. However, none of the species tested showed bands attributed to FESFPS primers in either the triplex (Fig. 3) or the FESFPS monosystem (data not shown).

The rhesus- $M$ and rhesus- $C$ samples only showed amplified products for the HPRTB system but not for the FESFPS or vWF primers. The rhesus HPRTB bands were within and above

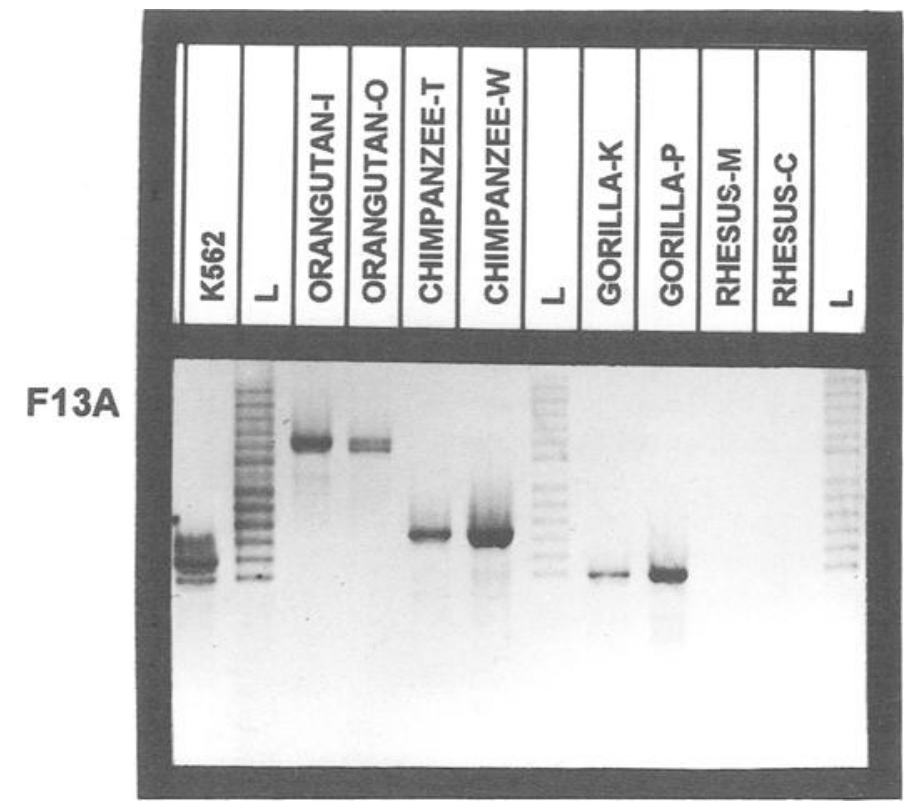

FIG. 4-Amplification and polyacnlamide gel electrophoresis of primate DNA samples using the GenePrint F13A01 monoplex system. DNA samples from the species indicated above were amplified as described in the Materials and Methods. Lanes labeled " $L$ " contain the F13AOI allelic ladder. The closed arrow indicates HPRTB amplified fragments well above the $A L$.

the allelic ladder (Fig. 3) which was verified using the monosystems for this loci (data not shown). Orangutan DNAs amplified with HPRTB primers although orangutan-I always amplified very weakly or not at all (Fig. 3). Further, there were weak bands generated by the VWF primers below the vWF allelic ladder for this species which could not be assigned an allele designation.

Both the chimpanzee and gorilla DNAs showed amplified fragments within the HPRTB ladder (Fig. 3), which were verified using the monosystem (data not shown). Chimpanzee-T and $-W$ had the HPRTB alleles " 12,13 " and " 11 ," respectively. Gorilla-K had the HPRTB alleles " 11,12 " and gorilla-P amplified a fragment adjacent to the "13" allele. The vWF primers amplified chimpanzee and gorilla DNA although the fragments migrated between the vWF allelic ladder fragments.

The F13AOl primers did not amplify any of the seventeen STR negative species mentioned above nor did the rhesus DNA samples show amplified fragments (Fig. 4). There were F13AO1 amplified products detected in the orangutan, chimpanzee and gorilla DNAs. A single band was evident for each of the species allowing for the following allelic designations: orangutans " 12 ," chimpanzee "6" and gorilla "4." Minor bands and smearing are occassionally detected with the HPRTB, vWF and F13A1 primer systems. This is most likely due to the high template concentrations used for these studies.

Table 2 is a summary of primate STR amplified products detected in these studies. The F13A01 STR primers amplified monomorphic fragments for all primates except the Rhesus samples which were negative. The CSF1PO, TPOX, THO1, HPRTB, and vWF STR primers showed single and double band patterns depending on the primate species. The FESFPS primers were the only human derived STR primers in which only human PCR products were detected. These preliminary data suggest that the 
locus F13A01 might be used to distinguish orangutan, chimpanzee, and gorilla as their amplified product patterns differ from one another, but pairs of individuals within a single species display identical patterns. Inbreeding of monkey colonies could also explain this result.

Tandem repeat arrays are known to exist in most, if not all, eukaryotic genomes [1]. The purpose of this study was to determine if amplified fragments are obtained from animal species DNA using human STR systems and if so, what is the nature of the band pattern compared to human DNA. We have demonstrated that the nine STR systems analyzed for this study are generally primate specific and do not amplify DNAs from any of the nonprimate species tested. It would be interesting to sequence the DNA fragments that have been amplified using these STR systems to determine their repeat array.

\section{References}

[1] Charlesworth, B., Sniegowski, P., and Stephan, W., "The Evolutionary Dynamics of Repetitive DNA in Eukaryotes," Nature, Vol. 371, 1994, pp. 215-220.

[2] Tautz, D., "Hypervariability of Simple Sequences as a General Source for Polymorphic DNA Markers," Nucleic Acids Research, Vol. 17, 1991, pp. 6463-6471.

[3] Puers, C., Hammond, H., Jin, L., Caskey T., and Schumm, J., "Identification of Repeat Sequence Heterogeneity at the Polymorphic Short Tandem Repeat Locus HUMTHOI [AATG]n and Reassignment of Alleles in Population Analysis by Using Locus Specific Allelic Ladder," American Journal of Human Genetics, Vol. 53, 1993, pp. 953-958.

[4] Polymeropoulos, M., Wrath, D., Xiao H., and Meril, C., "Tetranucleotide Repeat Polymorphism at the Human Coagulation Factor XII A Subunit Gene (F13A1)," Nucleic Acids Research, Vol. 19, p. 4306.

[5] Edwards, A., Civitello, A., Hammond, H., and Caskey, C., "DNA
Typing and Genetic Mapping with Trimeric and Tetrametric Tandem Repeats," American Journal of Human Genetics, Vol. 49, 1991, pp. 746-756.

[6] Edwards, A., Civitello, A., Hammond, H., Caskey, C., and Chakraborty, R., "Genetic Variation at Five Trimeric and Tetrameric Tandem Repeat Loci in Four Human Population Groups," Genomics, Vol. 12, 1992, pp. 241-253.

[7] Hammond, H., Jin, Y., Zhong, Y., Caskey, C., and Chakraborty, R., "Evaluation of 13 Short Tandem Repeat Loci for Use in Personal Identification Applications," American Joumal of Human Genetics, Vol. 55, 1994, pp. 175-189.

[8] "Guidelines for a Quality Assurance Program for DNA Analysis," Crime Laboratory Digest, Vol. 18, 1991, pp. 44-75.

[9] Committee on DNA Technology in Forensic Science, The National Research Council, DNA Technology in Forensic Science, National Academy Press, Washington, D.C., 1992.

[10] Maniatis, T., Fritsch, E., and Sambrook, J., Molecular Cloning: A Laboratory Manual, Chapter 9, Cold Spring Harbor Laboratory, Cold Spring Harbor, NY, 1982, p. 269.

[11] Edwards, A., Voss H., Civitello, A., Stegemann, J., Schwager, C., Zimmermann, J., et al., "Automated DNA Sequencing of the Human HPRTB Locus," Genomics, Vol. 6, 1990, pp. 593-608.

[13] Hearne, C. and Todd, J., "Tetranucleotide Repeat Polymorphism at the HPRT Locus," Nucleic Acids Research, Vol. 19, 1991, p. 5450.

[14] Jeffreys, A., Royle, N., Wilson, V., and Wong, Z., "Spontaneous Mutation Rates to New Length Alleles at Tandem-Repetitive Hypervariable Loci in Human DNA," Nature, Vol. 332, 1988, pp. 278-281.

[15] Jeffreys, A., Wilson, V., and Thein, S., "Hypervariable 'Mini-Satellite' Regions in Human DNA," Nature, Vol. 314, 1985, pp. 67-73.

[16] Patel, P., Nussbaum, R., Gramson, P., Ledbetter, D., Caskey, C., and Chinault, A., "Organization of the HPRT Gene and Related Sequences in the Human Genome," Somatin Cell Molecular Genetics, Vol. 10 , 1984, pp. 483-493.

Address requests for reprints or additional information to Cecelia Crouse, Ph.D.

Palm Beach County Sheriff's Office Crime Lab

3228 Gun Club Rd.

West Palm Beach, FL 33406 